\title{
A simple innovative Chennai handshake technique for reducing anterior dislocation of shoulder joint: a community study of 60 cases
}

\author{
Muhammad Thahir $^{1 *}$, B. Suresh Gandhi ${ }^{2}$, Kalaivanan Kanniyan ${ }^{3}$, Shanmugasundaram ${ }^{4}$
}

\author{
${ }^{1}$ Department of Orthopaedic Surgery, Dubai Hospital, Dubai \\ Department of Orthopaedic Surgery, ${ }^{2}$ JIPMER, Pondicherry; ${ }^{3}$ SRM Institute of Medical Sciences, Chennai; ${ }^{4}$ Saveetha \\ Medical College, Chennai, Tamil Nadu, India
}

Received: 01 October 2017

Revised: 12 October 2017

Accepted: 14 October 2017

\section{*Correspondence: \\ Dr. Muhammad Thahir, \\ E-mail: thahir23@gmail.com}

Copyright: ( $)$ the author(s), publisher and licensee Medip Academy. This is an open-access article distributed under the terms of the Creative Commons Attribution Non-Commercial License, which permits unrestricted non-commercial use, distribution, and reproduction in any medium, provided the original work is properly cited.

\section{ABSTRACT}

Background: Anterior shoulder dislocation is one of the commonest dislocation of our body. We here by introduce a simple, safe, successful and easily reproducible "Chennai Handshake Technique" to relocate anterior dislocation of shoulder joint.

Methods: This method was performed by a single surgeon on sixty cases (51 males, 9 females) of anterior shoulder dislocation with a mean age of 38 years (18 to 58 years) between 2008 to 2013 in tertiary care center. Among sixty patients, there were 12 cases (20\%) of fresh dislocation and 48 cases (80\%) of recurrent dislocation. It comprises of holding the hand in a classical double shake position and then giving a gentle longitudinal traction (stage I) followed with slow abduction and external rotation of arm (stage II), thus reducing the shoulder dislocation. Vertical oscillation may be a part of stage II in some cases before the external rotation.

Results: The index time of patient presenting to the casualty ranged from one hour to $18 \mathrm{hrs}$ with a mean of $4.45 \mathrm{hrs}$. The reduction time ranged from 1 to 16 min with a mean of $5.6 \mathrm{~min} .17$ dislocations (28.3\%) were reduced during stage I. 41 dislocations $(68.3 \%)$ were reduced during stage II. 5 dislocations $(12.1 \%)$ needed vertical oscillation during stage II. we failed in two cases (3.3\%).

Conclusions: No need of assistant, no need of anesthesia, no complications makes this technique, a more look back one.

Keywords: Anterior shoulder dislocation, Chennai handshake technique

\section{INTRODUCTION}

The shoulder joint is a type of ball and socket joint with greater mobility at the expense of its stability. Shoulder dislocates either in anterior, posterior or inferior direction with anterior dislocation as the commonest type accounting for nearly $96 \%{ }^{1}$ Anterior dislocation is further sub-divided into sub coracoid, sub glenoid, sub clavicular and intrathoracic types with sub coracoid as the commonest. ${ }^{2}$ The mode of injury is mostly by a fall onto an outstretched arm or by direct force over the posterior aspect of humeral head. The mechanism of injury is the indirect leverage of humeral head anteriorly over the shoulder joint with some combination of abduction, extension and external rotation. ${ }^{3}$

Kocher's method has been one among the commonest method used to relocate the shoulder dislocation but is no longer recommended due to high incidence of complications. Neuro-vascular injuries (axillary artery, brachial plexus) have been reported along with ligament injuries (rotator cuff, glenohumeral ligaments) and bony 
injuries (Bankart lesion in glenoid, Hill-sach's lesion of humeral head, humeral shaft and neck fractures) with the old reduction techniques. ${ }^{4-8,16}$

The traditional techniques such as Hippocrates, Stimson, Spaso, Eskimos and Kocher, Milch, scapular manipulation and combination of these techniques. Maneuver which mainly use traction, counter traction and leverage increases muscle spasm and make the reduction difficult.

\section{METHODS}

The prospective study was conducted between January 2010 to December 2013 by a single surgeon in tertiary care center. This study was examined and approved by the human research ethics committee. The patient with acute anterior glenohumeral dislocation, patient with recurrent anterior glenohumeral dislocation and the patient with acute anterior glenohumeral dislocation with/without greater tuberosity fracture were included in the study.

The patient with known ischemic heart disease, neurovascular deficit of the injured limb, obesity, old age, polytrauma/unconscious status, posterior glenohumeral dislocation and non-cooperative patients were excluded from the study. Total of sixty cases (51 males and 9 females) of anterior shoulder dislocation with a mean age of 38 years (18 to 58 years) included in the study. Among the sixty there were 12 cases $(20 \%)$ of fresh dislocation and 48 cases $(80 \%)$ of recurrent dislocation.

All the patients were thoroughly examined to rule out any other injuries and comorbid conditions especially ischemic heart disease. Patients were taken for immediate bedside reduction. We explained the procedure and the consent of the patient was taken. The patient was lied down on a couch in a comfortable manner.

\section{Stage I}

Hold the patient's hand in a classical hand shake position and start giving longitudinal traction along the axis of the limb. The traction should be slow, gradual and sustained. Wait for ten to twenty minutes, patiently without doing any other manipulation. Most of the dislocations get reduced with this alone.

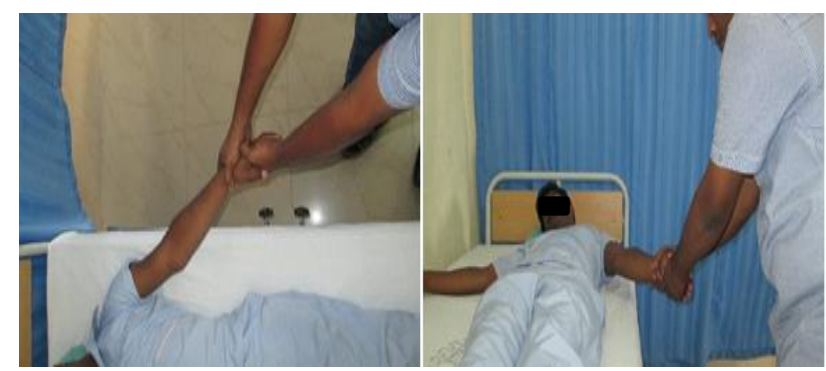

Figure 1: Stage-I.

\section{Stage II}

If reduction is not achieved with longitudinal traction alone, it is then followed with slow abduction of arm maintaining the longitudinal traction. Then abduction given ranges from 900 to 1200 . Sometimes, vertical oscillation is also required during the stage II to relax the muscles around shoulder joint. Make sure the patient is comfortable and allows their arm to fall free without any muscle spasm.

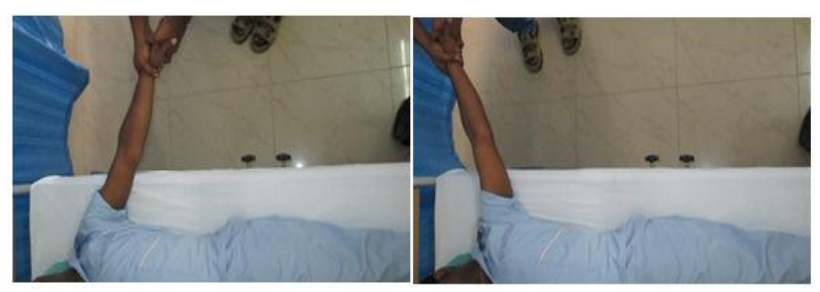

Figure 2: Stage-II. Slow abduction of arm maintaining the longitudinal traction.

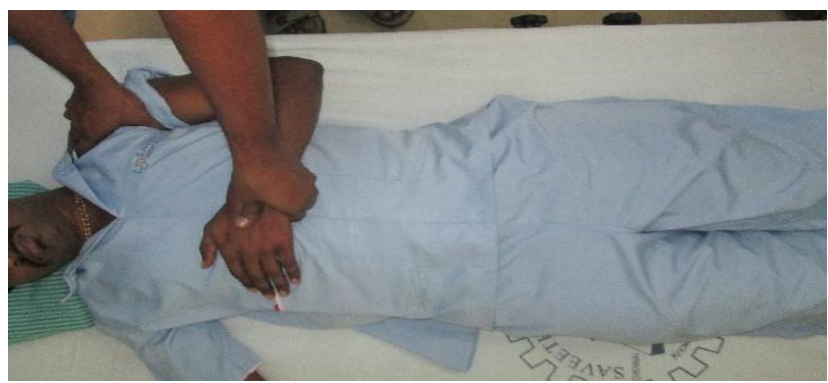

Figure 3: Internal rotation of the arm-reduced.

\section{RESULTS}

There were 51 male and 9 female patients in our study group. The age group ranged from 18 to 58 years with a mean age of 38 years. Right shoulder (38) was involved in mostly, left shoulder 22 were in number. There were 12 cases of fresh dislocation and 48 cases of recurrent dislocation. The reduction time ranged from 1 to $16 \mathrm{~min}$ with a mean of $5.63793 \mathrm{~min}$. There were two cases of dislocation with GT fracture.

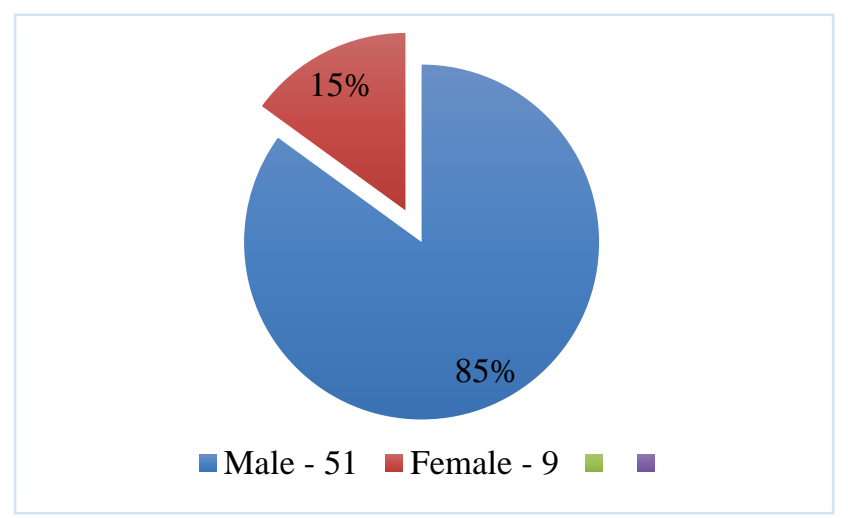

Figure 4: Sex distribution. 


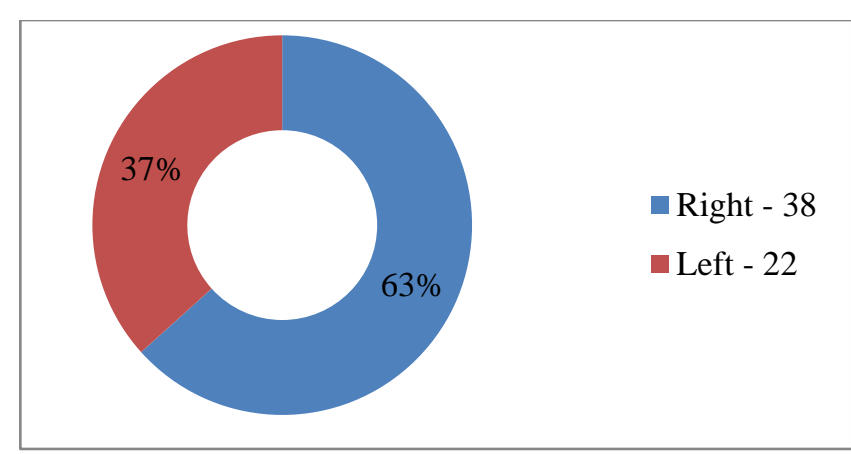

Figure 5: Side distribution.

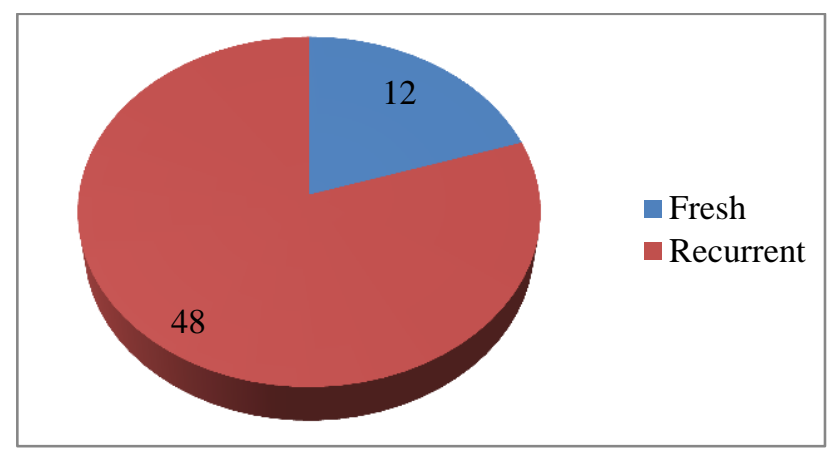

Figure 6: Episode of dislocation.

The index time of patient presenting to the casualty ranged from one hour to $18 \mathrm{hrs}$ with a mean of $4.45 \mathrm{hrs}$. 18 dislocations were reduced during stage I. 42 dislocations were reduced during stage II. 5 dislocations needed vertical oscillation during stage II. We were not able to relocate the joint in two cases and were finally reduced under general anesthesia. Three of our patients had history of diabetes mellitus and one case had history of ischemic heart disease.

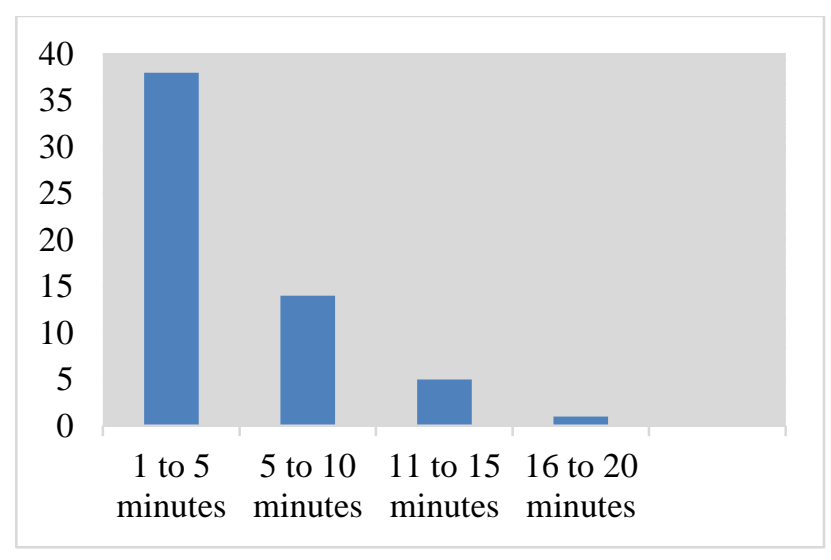

Figure 7: Reduction time of the patient.

Table 1: Reduction time.

\begin{tabular}{|l|l|}
\hline No of patients reduced & $\mathbf{5 8}$ \\
\hline Mean Average & 5.64 \\
\hline Mode & 5 \\
\hline Range of time & 1 to 16 Minutes \\
\hline
\end{tabular}

\section{DISCUSSION}

The shoulder joint is the commonest joint to get dislocated with an incidence of 8.2 to 23.9 per $1,00,000$ person/year. Shoulder dislocation account for $50 \%$ of all dislocations and $90-98 \%$ of them are anterior dislocation. Main etiology being trauma. It's common among young male athletes. ${ }^{1-3}$

Hitherto, there have been a various reduction technique described in literature. Kocher's Method, Milch technique, Hippocratic method, Matsen's traction counteraction, Spaso technique, Eskimo technique, Manes method, Scapular manipulation, Stimpson, FARES are some of the named reduction techniques. ${ }^{4-9,15}$

Further there is a lack of proper randomized studies to label any single technique as gold standard.

So there has been an evolution in inventing a simple, safe, successful and easily reproducible method to relocate anterior shoulder dislocation with less pain, less hospital stay and cost effective to the patient.

FARES technique presents with good success rate of $88.7 \%$, time of reduction of 2.36 minutes and visual analogue scale (VAS) score of 1.57 when compared with the efficacy of Kocher's method $68 \%$ (34) and Hippocratic method $72.5 \%$ (37) and reduction time of 4.32 with Kocher method and 5.55 with Hippocratic method and a VAS score of 1.57 with FARES method, 5.44 with Kocher and 4.88 with Hippocratic method. RCT parallel-group single center study. ${ }^{11,15}$

Tsoi et al, showed successful FARES technique in 6 out 9 cases with reduction time ranging from 10 seconds to 2 minutes and successful Spaso technique in 2 out of 9 cases with an average reduction time of 20 seconds. ${ }^{10}$

Mario et al used ERM external rotation method in their study group of 31 patients. The average reduction time was $3 \mathrm{~min}$ with nil complication. Sedation was required in 6 patients to achieve reduction. ${ }^{12}$

Miljesic et al study showed Spaso technique was successful in 14 of 16 cases with an overall success rate was $87.5 \%$ with no complications. ${ }^{13}$

Scapular manipulation technique have also been reported with a good reproducible success rate of $87.5 \%$ (McNamara et al) and 90.2\% (Baykal et al) without any sedative in first attempt. ${ }^{7,14}$

Among methods that use counter support on the axilla, the most widely known are variants of the Hippocratic method, which use a sheet (Matsen method), the operator's fist or foot (Oribase technique with the heel in the axilla, now discarded), or the back of a chair. ${ }^{15}$ 
Intraarticular lignocaine usage in early presentations and intravenous anesthesia for late ( $>5$ hrs.) presentations have been widely recommended for pain free shoulder relocation. But still some complications have been reported with such usage of local and general anesthetic agents.

Immobilization followed by shoulder physiotherapy have been advised to all the patients based on their age, mode of injury and either it's a fresh or recurrent. Usually the fresh trauma induced shoulder dislocation have been advised a period of three weeks immobilization for the anterior tissues to scar to avoid recurrence and then followed with a brief period of rehabilitation protocol. ${ }^{15}$

The ideal reduction technique of relocating shoulder joint with minimal traction, nil rotational force, no assistance and less patient interference is in search Denovo.

\section{Strength of the study}

- Easily reproducible.

- Paramedical staffs/emergency mobile team.

\section{Limitations of the study}

Single ethnic group.

\section{CONCLUSION}

Reduction of anterior shoulder dislocation by "Chennai handshake technique" yields high success rate in a quick time with less discomfort to the patients. This technique is easy to learn and simple to practice in emergency department even in patient with recurrent anterior glenohumeral dislocation and acute anterior glenohumeral dislocation with or without greater tuberosity fracture without pre medications, with no fear of significant complications.

\section{Funding: No funding sources}

Conflict of interest: None declared

Ethical approval: The study was approved by the institutional ethics committee

\section{REFERENCES}

1. Blake R, Hoffman J. Emergency department evaluation and treatment of the shoulder and humerus. Emerg Med Clin North Am. 1999;17:85976.

2. Krøner K1, Lind T, Jensen J. The epidemiology of shoulder dislocations. Arch Orthop Trauma Surg. 1989;108:288-90.
3. Rockwood CA Jr, Wirth MA. Subluxation and dislocations about the G-H joint. In: Rockwood CA, Green DP, Burkolz RW, Heckman JD, editors. Rockwood and Green's fractures in adult. 4th ed. Philadelphia: Lippincott-Raven Publishers; 1996: 1306-1309.

4. Beattie TF, Steedman DJ, McGowan, Robertson CE. A comparison of the Milch and Kocher techniques for acute anterior dislocation of the shoulder. Injury. 1986;17:349-52.

5. Amar E, Maman E, Khashan M, Kauffman E, Rath E, Chechik O. Milch versus Stimson technique for nonsedated reduction of anterior shoulder dislocation: a prospective randomized trial and analysis of factors affecting success. J Shoulder Elbow Surg. 2012;21:1443-9.

6. Milch H. Treatment of dislocation of the shoulder. Surgery. 1938;3:732-40.

7. McNamara RM. Reduction of anterior shoulder dislocations by scapular manipulation. Ann Emerg Med. 1993;22:1140-4.

8. Kothari RU, Dronen SC. Prospective evaluation of the scapular manipulation technique in reducing anterior shoulder dislocation. Ann Emerg Med. 1992;21:1349-52.

9. Boss A, Holzach P, Matter P. A new selfrepositioning technique for fresh anterior-lower shoulder dislocation. Helv Chir Acta. 1993;60:2635.

10. Tsoi LCH, Wong MCK. FARES method to reduce acute anterior shoulder dislocation: a case series and efficacy analysis. Hong Kong $\mathrm{J}$ Emergency Med. 2012;19:65-9.

11. Sayegh FE, Kenanidis EI, Papavasiliou KA, Potoupnis ME, Kirkos JM, Kapetanos GA. Reduction of acute anterior dislocations: a prospective randomized study comparing a new technique with the Hippocratic and Kochermethods. J Bone Jt Surg Am. 2009;91(12):2775-82.

12. Marinelli M, Luigi de Palma. The external rotation method for reduction of acute anterior shoulder dislocations, J Orthop Traumatol. 2009;10(1):1720.

13. Miljesic S, Kelly AM. Reduction of anterior dislocation of the shoulder: the Spaso technique. Emerg Med. 1998;10(3/4):173-5.

14. Baykal B, Sener S, Turkan H. Scapular manipulation technique for reduction of traumatic anterior shoulder dislocations: experiences of an academic emergency department: Emerg Med J. 2005; 22(5):336-8.

15. Cunningham NJ. Techniques for reduction of anteroinferior shoulder dislocation. Emerge Med Australas. 2005;17:463-71.

Cite this article as: Thahir M, Suresh Gandhi B, Kanniyan K, Shanmugasundaram. A simple innovative Chennai handshake technique for reducing anterior dislocation of shoulder joint: a community study of 60 cases. Int J Res Orthop 2017;3:1084-7. 